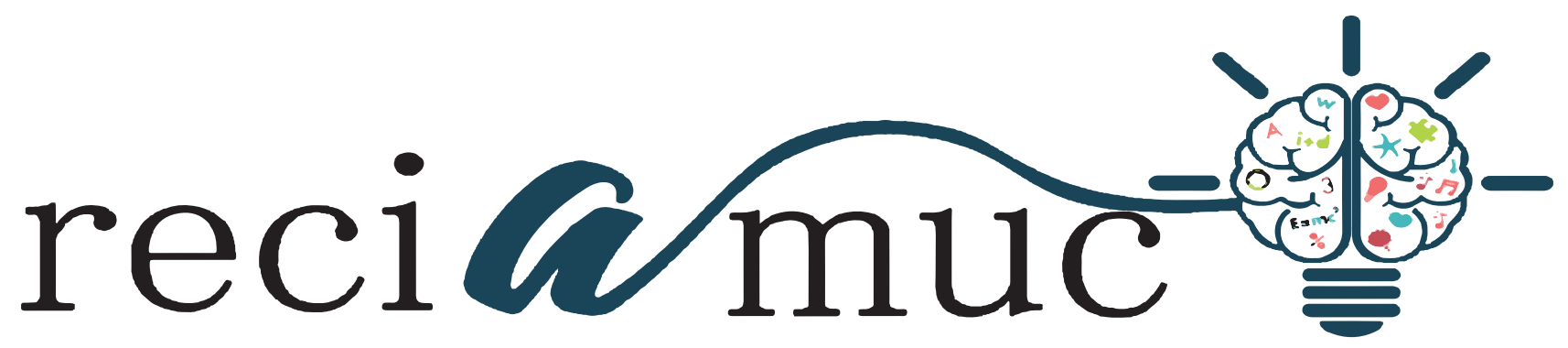

DOI: $10.26820 / \mathrm{reciamuc} / 4 .(1)$.enero.2020.49-58

URL: https://reciamuc.com/index.php/RECIAMUC/article/view/430

EDITORIAL: Saberes del Conocimiento

REVISTA: RECIAMUC

ISSN: $2588-0748$

TIPO DE INVESTIGACIÓN: Artículo de Revisión

CÓDIGO UNESCO: 3213.13 Estomatología

PAGINAS: $49-58$

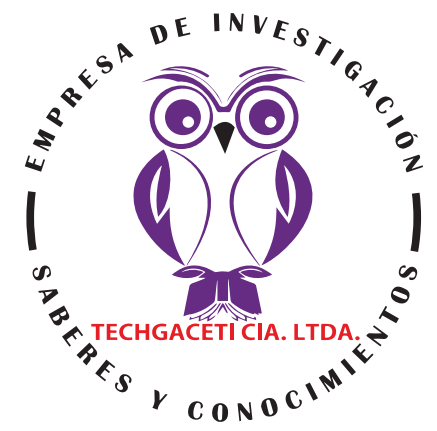

\title{
El Bruxismo, Conocimientos Actuales. Una Revisión de la Literatura
}

\section{Bruxism, Current Knowledge. A Review of the Literature. \\ Bruxismo, Conhecimento Atual. Uma revisão da literatura}

Patricia Judith Pinos Robalino'; Efigenia Monserrate Gonzabay Bravo2; María Jacqueline Cedeño Delgado $^{3}$

RECIBIDO: 18/11/2019 ACEPTADO: 20/12/2019 PUBLICADO: 31/01/2020

1. Magister en Diseño Curricular; Doctora en Odontología; Universidad de Guayaquil; Guayaquil, Ecuador; patricia.pinosr@ug.edu.ec; (D) https://orcid.org/0000-0001-7170-9381

2. Magister en Docencia Universitaria e Investigacion Educativa; Diploma Superior en Preparacion de Multirradiculares; Doctora en Odontología; Universidad de Guayaquil; Guayaquil, Ecuador; efigenia.gonzabayb@ ug.edu.ec; (D) https://orcid.org/0000-0001-5753-7747

3. Magister en docencia Univesitaria e Investigación Educativa; Doctora en Odontología; Universidad de Guayaquil; Guayaquil, Ecuador; maria.cedenod@ug.edu.ec; (D) https://orcid.org/0000-0002-9865-0938

\section{CORRESPONDENCIA}

Patricia Judith Pinos Robalino

patricia.pinosr@ug.edu.ec

Guayaquil, Ecuador 


\section{RESUMEN}

La salud bucodental está directamente relacionada con el goce de una buena salud y calidad de vida de las personas, resulta de gran importancia no sólo a nivel estético y social, sino también a nivel funcional. Uno de estos problemas estomatológicos que se presenta en todo el mundo es el Bruxismo, un hábito manifestado en el aparato masticatorio de importante observación en esta área por cuanto tiene como consecuencia cambios morfológicos que afectan la salud del paciente. La presente investigación se fundamenta en la revisión y compendio de los conocimientos relacionados con el bruxismo, desde la perspectiva de las innovaciones referidas a: Definición, Clasificación, Etiología, Diagnóstico, Lesiones Dentales y Tratamientos. El diseño de investigación que se llevó a cabo es de tipo documental o bibliográfico. El bruxismo es la actividad músculo-mandibular repetitiva caracterizada por apretamiento o rechinamiento de los dientes o por el apretamiento dental y empuje mandibular, pudiendo ocurrir durante la vigilia (bruxismo en vigilia) o durante el sueño (bruxismo del sueño). Es fundamental que los odontólogos se interesen cada día más en conocer los nuevos descubrimientos en la etiopatología y fisiopatología del bruxismo, con la finalidad de atacar el problema de raíz, tomando en consideración que el bruxismo es un síntoma y que lo que se debe tratar en realidad es la patología o condición que lo origina, en vez de enfocarse en decidir practicar tratamientos con miras a la restauración de los efectos o complicaciones del bruxismo.

Palabras clave: Bruxismo, Conocimientos, Estomatología, Bucodental, Rechinamiento.

\section{ABSTRACT}

Oral health is directly related to the enjoyment of good health and quality of life of people, it is of great importance not only at the aesthetic and social level, but also at the functional level. One of these stomatological problems that occurs worldwide is Bruxism, a habit manifested in the masticatory apparatus of important observation in this area because it results in morphological changes that affect the patient's health. This research is based on the review and summary of knowledge related to bruxism, from the perspective of innovations related to: Definition, Classification, Etiology, Diagnosis, Dental Injuries and Treatments. The research design that was carried out is documentary or bibliographic. Bruxism is the repetitive muscle-mandibular activity characterized by clenching or grinding of the teeth or by dental clenching and mandibular thrust, which can occur during wakefulness (wakefulness bruxism) or during sleep (sleep bruxism). It is essential that dentists become increasingly interested in knowing the new discoveries in the pathophysiology and pathophysiology of bruxism, in order to attack the root problem, taking into account that bruxism is a symptom and that what should be treated in reality is the pathology or condition that originates it, instead of focusing on deciding to practice treatments with a view to restoring the effects or complications of bruxism.

Keywords: Bruxism, Knowledge, Stomatology, Oral, Grinding.

\section{RESUMO}

A saúde bucal está diretamente relacionada ao gozo de boa saúde e qualidade de vida das pessoas, sendo de grande importância não apenas no nível estético e social, mas também no nível funcional. Um desses problemas estomatológicos que ocorre em todo o mundo é o bruxismo, um hábito manifestado no aparelho mastigatório de observação importante nessa área, pois resulta em alterações morfológicas que afetam a saúde do paciente. Esta pesquisa baseia-se na revisão e resumo de conhecimentos relacionados ao bruxismo, na perspectiva de inovações relacionadas a: Definição, Classificação, Etiologia, Diagnóstico, Lesões Dentárias e Tratamentos. O desenho da pesquisa realizada é documental ou bibliográfico. O bruxismo é a atividade músculo-mandibular repetitiva caracterizada por cerramento ou ranger dos dentes ou por aperto dentário e impulso mandibular, que podem ocorrer durante a vigília (bruxismo da vigília) ou durante o sono (bruxismo do sono). É essencial que os dentistas se interessem cada vez mais em conhecer as novas descobertas na fisiopatologia e fisiopatologia do bruxismo, a fim de atacar o problema radicular, levando em consideração que o bruxismo é um sintoma e que o que deve ser tratado na realidade é a patologia ou condição que o origina, em vez de se concentrar em decidir praticar tratamentos com o objetivo de restaurar os efeitos ou complicações do bruxismo.

Palavras-chave: Bruxismo, Conhecimento, Estomatologia, Oral, Moagem. 


\section{Introducción}

La salud bucodental está directamente relacionada con el goce de una buena salud y calidad de vida de las personas, resulta de gran importancia no sólo a nivel estético y social, sino también a nivel funcional dada su participación tanto en los procesos de comunicación (correcta pronunciación de las palabras) como en el proceso de inicio de digestión (masticación y deglución).

Uno de estos problemas estomatológicos que se presenta en todo el mundo es el Bruxismo, un hábito manifestado en el aparato masticatorio de importante observación en esta área por cuanto tiene como consecuencia cambios morfológicos que afectan la salud del paciente. En palabras más comunes el bruxismo consiste en apretar o rechinar los dientes y puede manifestarse durante la vigilia (bruxismo en vigilia) o durante el sueño (bruxismo del sueño). A pesar de que no se considera peligroso puede originar lo que se conoce como la triada del Bruxismo: lesiones dentales permanentes, dolores de cabeza o de oído y dolor en la mandíbula.

"El bruxismo puede erosionar y eventualmente desgastar el esmalte y la dentina en las coronas de los dientes, puede dañar coronas dentales metálicas o cerámicas, y hacer que los dientes se vuelvan móviles". La abrasión y la erosión ocasionadas por el bruxismo puede agravarse en pacientes con reflujo gastroesofágico y/o apnea obstructiva del sueño. (1)

Es importante destacar que, en la mayoría de los casos, el bruxismo no causa complicaciones graves, no obstante, el bruxismo intenso puede provocar:

- Daños a los dientes, las restauraciones, las coronas o la mandíbula.

- Dolores de cabeza relacionados con la tensión.

- Dolor de cuello o facial intenso.
- Trastornos que aparecen en las articulaciones temporomandibulares, que se encuentran justo delante de los oídos y que pueden sonar como un chasquido cuando se abre y se cierra la boca. (2)

Para Silva Contreras (3) la mayoría de las personas desconocen que padecen este hábito, razón por la cual su diagnóstico resulta sorprendente, incluso se pueden sentir avergonzados. Asimismo, se considera que pueden tomar años la aparición visible de los primeros signos de bruxismo. Algunos estudios plantean que el bruxismo es un modelo conductual extendido, en virtud de lo cual, se infiere que afecta a una parte significativa de la población mundial. (p. 58)

Romero, Torres, \& Correa (4) manifiestan al respecto que el bruxismo del sueño es un trastorno frecuente, presentando una prevalencia media de $8 \%$ aproximadamente en la población general. Igualmente, Existen algunas diferencias significativas relacionadas con la edad en que se presenta, "con rangos que van de un máximo del $40 \%$ en niños menores de 11 años, un 13\% en sujetos de entre 18-29 años y un descenso a niveles de hasta tan solo un $3 \%$ en individuos mayores de 60 años". En consecuencia, se puede concluir que la prevalencia presenta tendencia a disminuir con la edad. No obstante, estas cifras se deben interpretar con cautela, tomando en consideración que, a mayor edad, aumentan el número de pacientes con prótesis completa y con medicación, lo que se podría traducir en la supresión o disminución del bruxismo. (p. 7)

La presente investigación se fundamenta en la revisión y compendio de los conocimientos relacionados con el bruxismo, desde la perspectiva de las innovaciones referidas a: Definición, Clasificación, Etiología, Diagnóstico, Lesiones Dentales y Tratamientos.

\section{Materiales y métodos}

La presente investigación se desarrolló mediante el uso de computadores personales conectados a internet, en donde se buscó

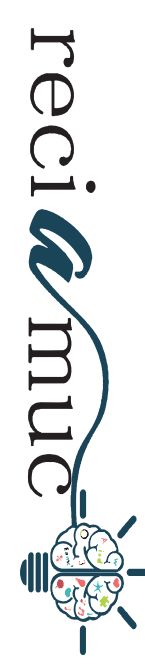


I material bibliográfico digitalizado a los fines de exponer acerca de los conocimientos actuales relacionados con el bruxismo, en razón de lo que la presente investigación se clasifica como una investigación de tipo documental o bibliográfica. Asimismo, el material se localizó a través de las bases de diversas bases de datos y páginas web reconocidas en el área de la salud en general, entre las que figuraron: MedlinePlus, Manual MSD, PubMed, SciELO, Dialnet, entre otras

Para la búsqueda se utilizaron los siguientes descriptores: "Bruxismo", "Tratamiento de Bruxismo"; "Clasificación del Sueño" y "Tratamiento del Bruxismo", lo que resultó en un aproximado de más de un centenar de miles de registros bibliográficos que se fueron filtrando bajo los criterios de idioma español, relevancia, correlación temática y actualidad (fecha de publicación en los últimos seis años).

\section{Resultados}

\section{Definición}

La tercera edición del texto clínico autorizado para el diagnóstico de los trastornos del sueño (International Classification of Sleep Disorders - Third edition) 2014, citado por Fuentes Casanova (5), define al Bruxismo como "la actividad músculo-mandibular repetitiva caracterizada por apretamiento o rechinamiento de los dientes o por el apretamiento dental y empuje mandibular, pudiendo ocurrir durante la vigilia (bruxismo en vigilia) o durante el sueño (bruxismo del sueño)". (p. 182)

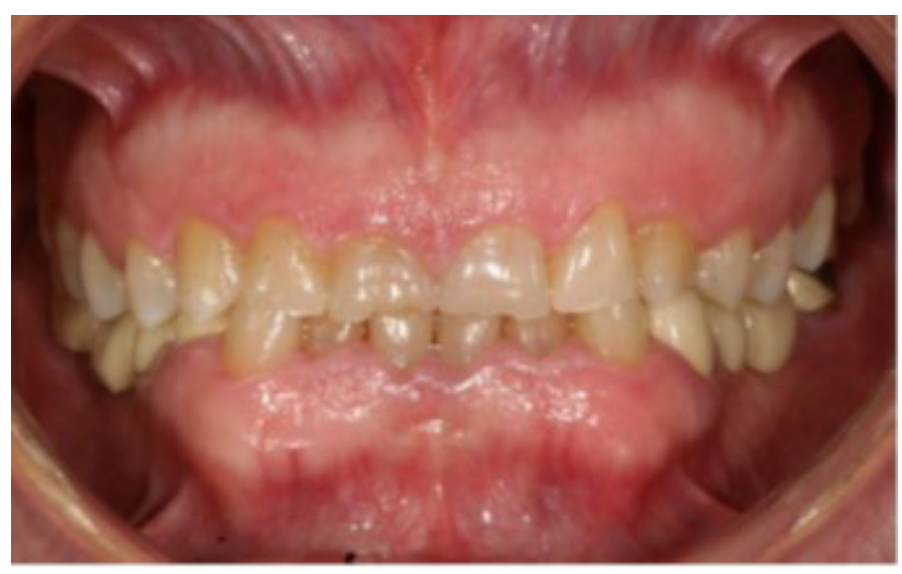

Figura 1. Desgaste dental por Bruxismo

Fuente: Romero, A.; Torres, J.M. y Correa, L, $2014^{4}$

\section{Clasificación}

Fleta Zaragozano (6) en su trabajo clasifica el bruxismo como primario y secundario. El bruxismo primario, también llamado idiopático es la manifestación del apretamiento diurno y aquel producido también durante el sueño, en aquellos casos donde se desconocen otros problemas o causas médicas. Por otra parte, el bruxismo secundario, también denominado iatrogénico, son formas de bruxismo asociados a problemas neurológicos, desórdenes del sueño, problemas psiquiátrico y administración de drogas. El bruxismo puede manifestarse durante el día (bruxismo diurno) o durante la noche (bruxismo nocturno). Es allí donde radica la importancia de señalar en el diagnóstico de bruxismo las circunstancias e identificar el tipo o clase, por cuanto de ello dependerá su tratamiento, por ejemplo, "el bruxismo nocturno, como una parasomnia, corresponde a una entidad que debe ser tratada de diferente manera al bruxismo diurno". (p. 486-e1) 


\section{Etiología}

La etiología del bruxismo en la actualidad sigue siendo motivo de debate científico, ya que esta manifestación a lo largo del tiempo se ha ido relacionando con variados factores de riesgo, en virtud de lo cual existe también variadas teorías al respecto. Se puede decir que hasta la fecha no se precisa del todo la causa del bruxismo, no obstante, se reconoce que el origen podría deberse a una combinación de factores físicos, psicológicos y genéticos.

Contreras, Contreras, \& Ovalle (7) en su estudio se plantearon identificar la asociación fonoaudiológica, psicológica y odontológica en la patología del bruxismo en los jóvenes universitarios de Cúcuta, Colombia, donde registraron que:

En la categorización prevalecen los síntomas somáticos en los pacientes estudiados; ya que se observa en los pacientes el desgaste de dientes, en otros pacientes hay presencia de carillas dentales, maloclusiones dentales, zumbido de oídos, dolores musculares; por lo tanto, los pacientes expresan en la historia clínica odontológica que son bruxómanos. Además de Predominan los síntomas psíquicos en los pacientes estudiados, a partir del diagnóstico clínico, se observa la ansiedad con características de preocupaciones, tensión, insomnio, y estrés. En tal sentido, concluyen que los estudiantes que presentan desórdenes témporomandibulares, requieren acceder a un tratamiento interdisciplinario (fonoaudiología, psicología y odontología); en la medida que la articulación témporomandibular influyen sobre los músculos de la laringe y los espacios neuromusculares que determinan la función de los resonadores; siendo alterada el habla en la persona en su motricidad orofacial; en este sentido es importante el rol del fonoaudiólogo en la intervención de la disfunción temporomandibular; por eso, los síntomas fonoaudiológicos generalmente están asociados a alteraciones de origen emocional. (p. 7)
Fuentes Casanova (5) clasifica estos factores de riesgo en dos grupos que pudieran ser interactuantes: factores periféricos (morfológicos/anatómicos) y centrales (psicológicos y fisiopatológicos):

Los factores periféricos (morfológicos o anatómicos) se refieren a las alteraciones dentoesqueléticas. Dentro de este grupo podemos mencionar las alteraciones en la oclusión dental y en las interrelaciones anatómicas de las estructuras óseas cráneo-cérvico-faciales, posibilidad cimentada en el asumir que, en ausencia de equilibrio oclusal por razones dentales o esqueléticas, producen activación de los receptores periodontales, con una respuesta muscular secundaria de tipo reactivo.... En la actualidad, los clínicos han ampliado la búsqueda de otros factores causales, donde la oclusión dentaria cede su accionar a otras áreas del conocimiento (factores psicológicos y fisiopatológicos), lo que demuestra una tendencia en investigación del bruxismo más cercana a un modelo/paradigma biomédico-biopsicosocial que únicamente a la oclusión. (p. 183)

En cuanto a los factores centrales (psicológicos y fisiopatológicos), se puede mencionar que el bruxismo en su fase de vigilia puede atribuirse a emociones tales como: ansiedad, estrés, ira, frustración o tensión. Asimismo, podría tratarse de "una estrategia de afrontamiento o un hábito durante la concentración profunda. El bruxismo durante el sueño puede deberse a la actividad de masticar relacionada con el sueño y asociada con despertarse durante el sueño".

Es importante mencionar entre los factores fisiopatológicos los trastornos del sueño, consumo de fármacos dopaminérgicos, alteraciones neuroquímicas, noradrenérgicos, serotoninérgicos, antidepresivos, GABAérgicos y psicotrópicos, enfermedades, traumatismos y factores genéticos. 


\section{Tipo de lesiones dentales producidas por} el bruxismo

González, Midobuche, \& Castellanos (8) entre las lesiones dentales ocasionadas por el bruxismo, menciona las más destacadas:

- Abfracciones. Son lesiones consideradas de origen multifactorial, siendo su principal etiología trauma deslizante o excéntrico en donde cargas de diversa intensidad, frecuencia, duración y dirección, inducen tensiones por flexión a través del diente; la resistencia a la flexión produce tensión y compresión a nivel del fulcro, el cual en periodontos intactos se encuentra cercano al nivel de la unión cemento-esmalte. (véase Figura 2)

- Atrición. Desgaste por fricción diente a diente que puede ocurrir durante la deglución con movimiento deslizante y apretamiento excéntrico. El desgaste se vuelve severo durante el bruxismo con evidencia de una rápida pérdida de sustancia dental. (véase Figura 3)

- Corrosión. Es la pérdida de superficie dental por acción química o electroquímica. Existen dos fuentes de corrosión. La endógena como la bulimia, reflujo gastroesofágico, líquido crevicular y caries. La otra es exógena, representada por alimentos, sustancias o bebidas con pH menor de 5.5, así como por medicamentos, drogas y abuso de alcohol.

- Abrasión. Se define como la fricción entre diente de un agente exógeno que provoca desgaste.

- Lesiones mixtas. Existe la posibilidad de que los mecanismos de daño y desgaste dental no sean procesos puros sino compuestos. (p. 94, 95)

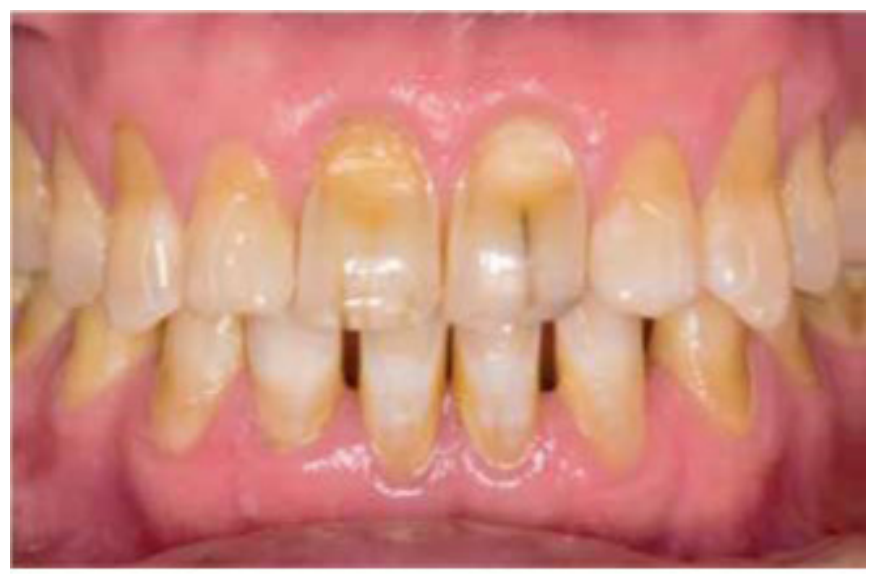

Figura 2. Presencia de abfracciones

Fuente: González, Midobuche, \& Castellanos, $2015^{2}$ 


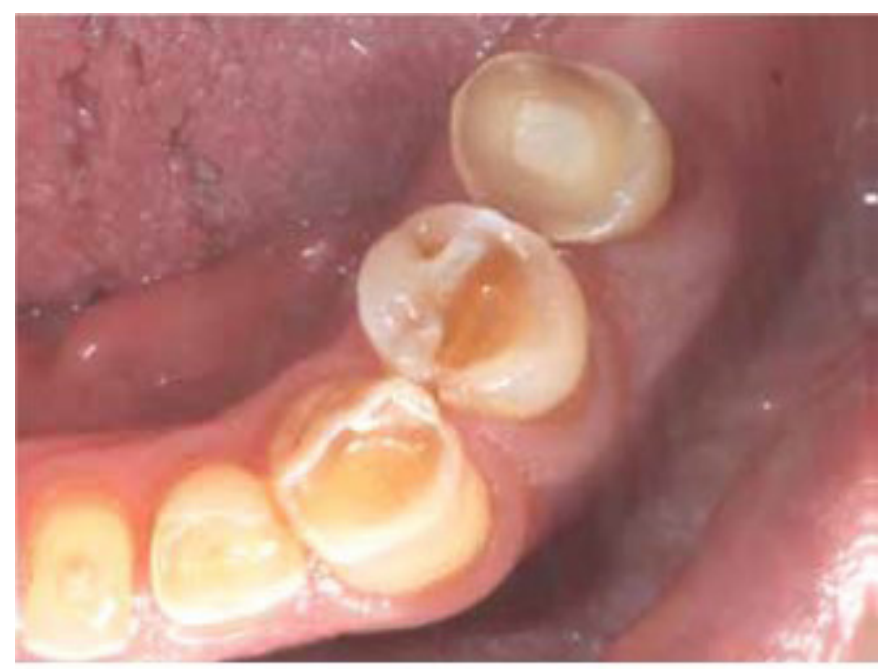

Figura 3. Ejemplo de atrición en bordes incisales y caras oclusales

Fuente: González, Midobuche, \& Castellanos, 2015²

\section{Diagnóstico}

Bruno \& Restrepo (9) resaltan que para cualquier diagnóstico resulta fundamental entender, que el bruxismo es un síntoma, en razón de lo cual, la evaluación debe estar orientada a evaluar los síntomas que lleven a un diagnóstico particular. En el caso del bruxismo del sueño, recomienda se lleve a cabo la evaluación del paciente de la siguiente manera:

- Anamnesis: evaluar calidad del sueño, alimentación, medicación, relato de los padres/familia sobre rechinamiento dental, quejas de dolores musculares, cefaleas.

- Evaluar dieta, rutinas de sueño y características de la vía aérea.

- Ficha de examen clínico: evaluar desgastes dentales atípicos, úlceras y elevaciones lineales en la mucosa yugal uni o bilateralmente.

- Evaluación de la respiración.

- Evaluación de Trastornos Temporomandibulares (TTM).

- Evaluación a través de polisomnografía, cuando se requiera de un diagnóstico preciso.
- Evaluación de catecolaminas en orina, cuando se requiera determinar asociaciones precisas del bruxismo, con trastornos psiquiátricos $u$ hormonales concomitantes. (p. 102)

Por otra parte, Romo F. citado por Venegas Aguilera (10) menciona, para la determinación entre paciente bruxista y no bruxista, el protocolo anamnésico-clínico para diagnosticar bruxismo de Díaz et. al. Dicho "protocolo consta de 5 criterios anamnésicos y 5 criterios clínicos. Los 10 criterios específicos que componen este protocolo son:

a) Criterios anamnésicos: 1. Reporte de apriete y/o rechinamiento dentario. 2. Historia reciente de apriete y/o rechinamiento dentario reportado por un amigo, pariente y/o esposo. 3. Dolor o sensación de cansancio en los músculos de la cara. 4. Reporte de pérdida o fractura de restauraciones, coronas, puentes u otros, sin una causa aparente. 5. Sensación de poseer los dientes desgastados. b) Criterios clínicos: 1. Dolor muscular a la palpación. 2. Presencia de bruxofacetas. 3. Hipertrofia del músculo masétero y/o temporal. 4. Movilidad dentaria aumentada asociada a facetas de desgaste dentario. 5. Pérdida o fractura de restauraciones sin una causa aparente. (p. 30) 
Dentro de los criterios principales se encuentran de los anamnésicos, el apriete y/o rechinamiento dentario, el resto de los criterios anamnésicos son considerados como secundarios. Para la valoración general, se cuentan los criterios, en donde, se considerará un paciente con bruxismo que cumpla con 3 criterios específicos, como mínimo, dentro de los cuales, 2 criterios deben corresponder al tipo anamnésicos, y de estos, uno debe ser principal y uno secundario, y 1 criterio de tipo clínico.

\section{Tratamiento}

El tratamiento del bruxismo debe considerarlo como una actividad parafuncional multifactorial, en tal sentido, este debe estar orientado básicamente a la identificación y control de los factores que lo están originando. Asimismo, debe ir en función de la compensación de los daños ocasionados por esa actividad.

\section{Bruxismo Nocturno (BN)}

En el caso del tratamiento del bruxismo nocturno (BN), Fernández, Delgado, \& Castellanos (11) resalta en su investigación:

El bruxismo nocturno puede ser producto de una desregulación pura del sistema nervioso autónomo, lo que implicará conocer y trabajar más sobre las sustancias neurotransmisoras que por su exceso o carencia pueden alterar los procesos del sueño. De otro lado, el BN puede ser magnificado por eventos psicológicos y la capacidad individual mostrada de resistencia al estrés durante los periodos de vigilar; estableciéndose una condición de influencia bidireccionalmente con el bruxismo diurno; este último requiriendo de atención psicológica o psiquiátrica según las manifestaciones clínicas observadas. Los mediadores químicos, como es el caso de los niveles de serotonina y la afinidad hacia sus receptores, son variables a lo largo del día, relacionados con el estado de alerta y el ánimo (ritmo circadiano); frecuentemente afectados por estrés, ansiedad, depresión y otras variedades psicológicas y psiquiátricas. Los niveles máximos de serotonina se encuentran durante el día y disminuyen por la noche, esto debido a que los receptores son más abundantes y afines durante la noche, consumiéndole con el fin de reducir el estrés y concebir el sueño. El sistema serotoninérgico juega un papel fundamental en la regulación del ciclo sueño-vigilia; la administración de serotonina produce inducción del sueño, ya que se comporta como un hipnótico natural, reduciendo los despertares nocturnos. Sin embargo, el déficit produce reducción del sueño REM sin alterar el sueño NREM. (p. 191)

\section{Abordajes odontológicos}

Con la finalidad de preservar o mejorar los dientes del efecto del bruxismo el odontólogo puede recomendar:

- Férulas y protectores bucales. Están diseñados para mantener los dientes separados para evitar el daño causado al apretar o rechinar los dientes. Pueden estar hechos de acrílico duro o de materiales blandos para ajustarse a los dientes superiores e inferiores.

- Corrección dental. En casos graves cuando el desgaste del diente ha provocado sensibilidad o incapacidad para masticar de manera adecuada-, el dentista puede tener que remodelar las superficies de masticación de los dientes o colocar coronas para reparar el daño. (2)

Es importante acotar que el tratamiento odontológico, muy a pesar de que evitan o corrigen el desgaste de los dientes, no detienen el bruxismo.

\section{Férulas}

Las férulas o protectores bucales pueden ser efectivos en "el tratamiento del rechinamiento y apretamiento de los dientes y de los trastornos de la articulación temporomandibular" (véase Figura 5). La férula bien ajustada ayuda en la minimización de los 
efectos del bruxismo como la pulverización de los dientes. Existen muchos tipos de estos protectores o férulas, algunas están diseñadas para encajar en los dientes superiores, otras en los dientes inferiores, según la necesidad del paciente. Su diseño puede mantener la mandíbula en una posición más relajada, que permita facilitar alguna otra función. Este tratamiento se puede combinar con inyecciones de bótox en los músculos de la mandíbula, los cuales también han mostrado que tienen éxito en el control de apretar o rechinar los dientes. (12)

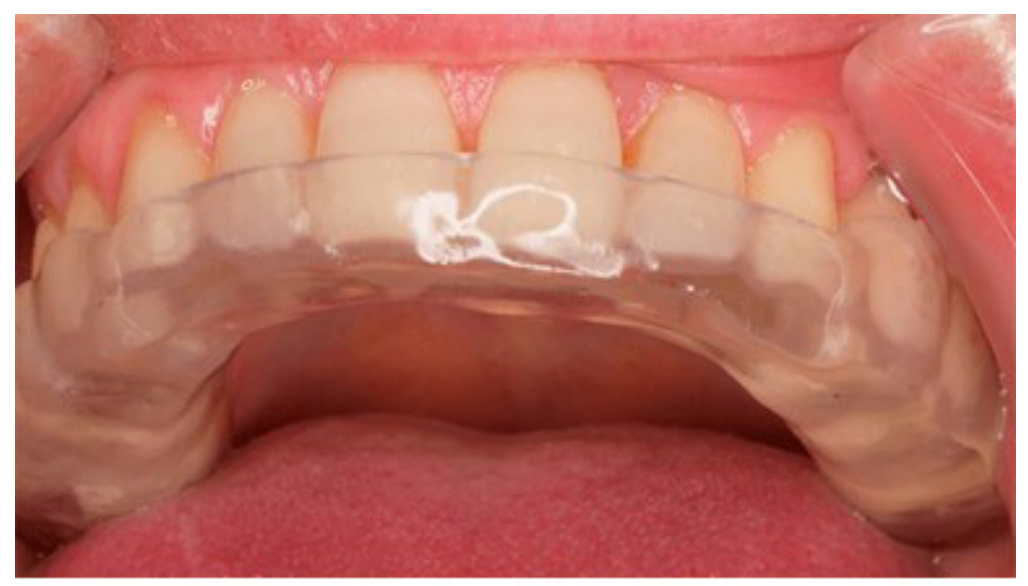

Figura 4. Férula oclusal

Fuente: Moradas, M. \& Álvarez, B, 2018 13

\section{Estilo de vida y remedios caseros}

Una alternativa en el tratamiento del bruxismo es mantener un estilo de vida sano, así como algunos remedios caseros. Estas medidas de cuidado personal pueden prevenir o colaborar en el tratamiento de esta condición, entre las que se pueden mencionar:

- Reduce el estrés. Escuchar música, tomar un baño caliente o hacer ejercicio son actividades que te pueden ayudar a relajarte y pueden reducir el riesgo de presentar bruxismo.

- Evita el consumo de sustancias estimulantes por la noche. No bebas café o té con cafeína después de la cena y evita el consumo de alcohol por la noche, dado que pueden empeorar el bruxismo.

- Mantén buenos hábitos de sueño. Dormir bien por la noche, lo que puede comprender el tratamiento de problemas de sueño, puede ayudar a reducir el bruxismo.

- Habla con tu pareja. Si duermes con tu pareja, pídele que preste atención a los sonidos de rechinamiento o chasquidos que podrías realizar al dormir para que puedas informárselos al dentista $\mathrm{O}$ al médico.

- Programa exámenes dentales periódicos. Los exámenes dentales son la mejor manera de identificar el bruxismo. El dentista puede advertir signos de bruxismo en la boca y en la mandíbula en los exámenes y las consultas regulares. (2)

\section{Conclusiones}

Es fundamental que los odontólogos se interesen cada día más en conocer los nuevos descubrimientos en la etiopatología y fisiopatología del bruxismo, con la finalidad de atacar el problema de raíz, tomando en consideración que el bruxismo es un síntoma y que lo que se debe tratar en realidad es la patología o condición que lo origina, en vez de enfocarse en decidir practicar tratamientos con miras a la restauración de los efectos o complicaciones del bruxismo.

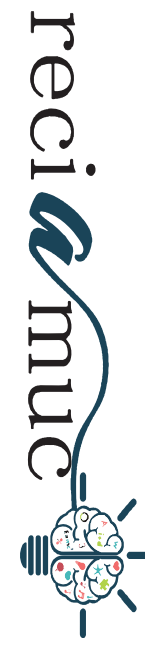


1 En este sentido, con una patogénesis precisa y la descripción de aquellos procesos que intervienen en los cambios morfofuncionales, el bruxismo puede ser diagnosticado y debidamente clasificado, en función de lo cual se puede establecer un tratamiento efectivo y eficaz, y un control que permita evaluar y reconsiderar los tratamientos aplicados en beneficio de los pacientes.

\section{Bibliografía}

1. Hennessy BJ. Manual MSD. [Online].; 2018 [cited 2020 Enero 10. Available from: https://www.msdmanuals.com/es-ve/professional/trastornos-odontol\%C3\%B3gicos/s\%C3\%ADntomas-de-los-trastornos-bucales-y-dentales/bruxismo.

2. Mayo Clinic. Mayo Clinic. [Online].; 2017 [cited 2020 Enero 05. Available from: https://www.mayoclinic.org/es-es/diseases-conditions/bruxism/ symptoms-causes/syc-20356095.

3. Silva Contreras AM. Bruxismo: su comportamiento en un área de salud. Revista de Ciencias Médicas. 2015; 19(1): p. 56-65.

4. Romero A, Torres JM, Correa L. BRUXISMO DEL SUEÑO. ACTUALIZACIÓN SOBRE MECANISMOS ETIOPATOGÉNICOS, DIAGNÓSTICO Y TRATAMIENTO. Revista de Neurología de la Sociedad Española de Sueño. 2014;: p. 185.

5. Fuentes Casanova FA. Conocimientos actuales para el entendimiento del bruxismo. Una revsión de la literatura. Revista ADM. 2018; 75(4): p. 180186.
6. Fleta Zaragozano J. Bruxismo en la infancia, causas y orientación terapéutica. Pediatría Integral. 2017; 21(7): p. 486.e1-486.e3.

7. Contreras L, Contreras R, Ovalle T. Asociación fonoaudiológica, psicológica y odontológica en la patología del bruxismoEn jóvenes universitarios de Cúcuta. Areté Fonoaudiología. 2019; 19(1): p. 8.

8. González EM, Midobuche EO, Castellanos JL. Bruxismo y desgaste dental. Revista ADM. 2015; 72(2): p. 92-98.

9. Bruno L, Restrepo C. Bruxismo del Sueño y Síndrome de Apnea Obstructiva del Sueño en Niños. Revisión narrativa. Revista de Odontopediatría Latinoamericana. 2016; 6(2): p. 99-107.

10. Venegas Aguilera MS. "ESTUDIO COMPARATIVO DE CALIDAD DE SUEÑO EN PACIENTES BRUXISTAS Y NO BRUXISTAS DE LA CLÍNICA ODONTOLÓGICA DE LA FACULTAD DE ODONTOLOGÍA UNIVERSIDAD DE CHILE AÑO 2012". Trabajo de Post Grado. Santiago: Universidad de Chile, FACULTAD DE ODONTOLOGÍA; 2013.

11. Fernández P, Delgado R, Castellanos JL. Alteraciones del sueño y bruxismo. revista ADM. 2018; 75(4): p. 187-195.

12. Enciclopedia Médica ADAM. MedlinePlus. [Online].; 2018 [cited 2020 enero 07. Available from: https://medlineplus.gov/spanish/ency/article/001413.htm.

13. Moradas M, Álvarez B. Actualización en la epidemiología y tratamiento multidisciplinar del Bruxismo: nuevos materiales. Revista Europea de Odontoestomatología. 2018.

\section{CitAR ESTE ARTICULO:}

Pinos Robalino, P., Gonzabay Bravo, E, \& Cedeño Delgado, M. (2020). El Bruxismo, Conocimientos Actuales. Una Revisión de la Literatura. RECIAMUC, 4(1), 49-58. doi:10.26820/reciamuc/4.(1).enero.2020.49-58

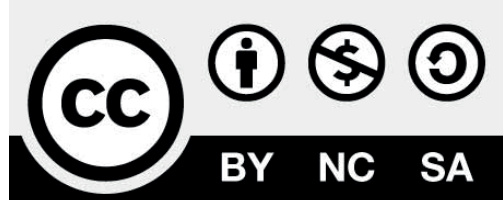

RECONOCIMIENTO-NOCOMERCIAL-COMPARTIRIGUAL CC BY-NC-SA

ESTA LICENCIA PERMITE A OTROS ENTREMEZCLAR, AJUSTARY CONSTRUIR A PARTIR DE SU OBRA CON FINES NO COMERCIALES, SIEMPRE Y CUANDO LE RECONOZCAN LA AUTORÍA Y SUS NUEVAS CREACIONES ESTÉN BAJO UNA LICENCIA CON LOS MISMOS TÉRMINOS. 